\title{
Morphological assessment of beef cattle according to carcass value
}

\author{
Jaime Alonso $^{(*)}$, Antonio Bahamonde ${ }^{(*)}$, Alfonso Villa ${ }^{(* *)}$, Ángel Rodríguez Castañón ${ }^{(* *)}$, \\ ${ }^{(*)}$ Artificial Intelligence Center (bww. aic.uniovi.es). University of Oviedo at Gijón. \\ Campus de Viesques, E-33271. Gijón (Asturias), Spain. \{jalonso, antonio\} @ aic.uniovi.es \\ ${ }^{(* *)}$ Association of Breeders of Asturiana de los Valles (ASEAVA). \\ Abarrio n 24. E-33424 Llanera (Asturias), Spain. \{avilla, arcastanon\} @ viaganadera.com
}

\begin{abstract}
In this paper we present an assessment function devised for bulls of a beef breed according to the specific market requirements of their carcasses. The procedure is completely repeatable since it is based on the measurements in centimeters of only 3 lengths of live animals plus an estimation of the curvature of the round profile. To derive this assessment, we used Artificial Intelligence tools based on Support Vector Machines (SVM). They allowed us to learn the estimations of the experts of the Association of Breeders (ASEAVA) about the value of carcasses of the animals in the sense that the score returned by the assessment function (on a scale 0-100) is higher for bulls with more valuable carcasses. Additionally, we studied the growth of bulls in order to adjust the assessments to a constant age of 365 days.
\end{abstract}

Keywords: Morphological assessment, Beef cattle, Carcass, Artificial Intelligence, Support Vector Machines (SVM)

\section{Introduction}

In this paper we present an assessment method devised for young bulls of a beef breed of the North of Spain, Asturiana de los Valles. This is a specialized breed with many double-muscled individuals; their carcass have dressing percentages over 60\%, with muscle content over 75\%, and with a low (8\%) percentage of fat (Piedrafita et al., 2003). The market target of these carcasses is made up of those consumers that prefer lean meat without any marbling (Díez et al., 2006; Díez et al., 2005; Del Coz et al. 2004).

It is generally acknowledged as a good practice the recording of live animal assessments; see for instance (ICAR 2005). It is recommended since assessments are description of an animal's morphology that reveals part of the animal's economic value. In this way, in addition to the description of a single animal, data from assessments can be used for evaluation of genetic selection of dairy, dual purpose and specialized beef breeds. The growth of the scores over years of selection for specific goals can be seen as a measure of the success of the selection policy. On the other hand, when the assessed traits are heritable, the scores can be directly used for selection purposes given that they are capturing part of animal's genetic value.

Usually, the assessment procedures are based on visual appreciations of well trained technicians that have to rank a number of morphological characteristics that include linear lengths of significant parts of animals' bodies. Although this process has been used successfully, it is clear that there is a problem with the repeatability of the assessments; not only between assessors, but even within assessors scoring the same animal in different times.

The assessment method described in this paper is completely repeatable and can be carried out using just 3 lengths (in centimeters) plus the appreciation of the curvature of the round profile (see curve $\mathbf{R P}$ in Figure 1). The method was designed to be used either by measuring manually the animals or by means of digital photographs. The purpose of the assessment is to evaluate the carcass economic value at a constant age. To compute the score achieved by each animal, we consider the muscularity as well as the body condition to return a single value or index that has a precise formal definition. The score of animals with higher estimations of carcass value at $\mathbf{3 6 5}$ days will be higher. The estimation of carcass values were learned from the estimations of the experts of the Association of Breeders (ASEAVA) according to the specific market requirements of Asturiana de los Valles. To adjust the scores to a fixed age, we found models of the growth of the assessments and of the morphological features required to compute the assessments. 
Given that this assessment is concerned with carcass value, it is possible to use it directly for selection. So, in (Nephawe et al. 2004) it is stated that some important characteristics related to carcass quality have high estimates of heritability $\left(\mathrm{h}^{2}\right)$ : hot carcass weight $(\mathbf{0 . 5 2})$, percentage of retail product $(\mathbf{0 . 5 9})$, longissimus muscle area (0.57). These results suggest that selection for carcass traits could be effective; see also (Koch et al., 2004).

The assessment method was developed using Artificial Intelligence tools. We learned the assessment formula from a dataset gathering the opinions of a set of experts of the Association of Breeders. We would like to emphasize that a core issue has been the way used to represent the opinion of the experts. Moreover, the selection of animals' measurements used to compose the assessment formula was not a human decision. The idea was to consider all reasonably possibilities for becoming inputs for the assessment formula. But we know that carrying out zoometric on an animal is a hard and risky task both for bulls and humans. Additionally, the presence of humans disturbs animals, what increase possible errors in measurements. Hence we needed to select carefully the set of measurements required for assessing. Thus, we employed a well-known algorithm used in Machine Learning to make a selection of features. In other words, we learned from the dataset of experts' preference judgments both the assessment formula and the set of variables required as inputs.

Notice that since we did not know the set of variables needed to induce an assessment function, we could not decide what measures had to be taken from each bull. Then, the use of digital photographs was especially useful, since we could reformulate the set of measurements. We started with two photos per animal showing the lateral and rear views (Figure 1), but we also tested the use of stereo photos taken from a zenithal view.

\section{Materials and methods}

\subsection{Data}

We collected and processed two sets of bulls. The first one was used to induce a formula able to assess the carcass performance using some morphological measurements. This set contains descriptions of 91 bulls of different ages of Asturiana de los Valles beef cattle breed. However, the representation of experts' assessments was a core point of our approach. The problem with a straightforward collection of numerical assessments given by a set of experts is that we observed that, as in many other cases (Del Coz et al., 2004), our experts tend to grade their preferences in a relative way, comparing animals with the other partners in the same batch. So, there is a kind of batch effect that biases their assessments: an animal surrounded by poor conformed cattle will probably obtain a higher score than if it were presented together with better bovines. From a computational point of view, this means that regression is not an acceptable method to induce an assessment function. Nevertheless, the knowledge of our experts can be reliably represented by means of orderings of small groups of animals according to experts' estimation of carcass values. We call preference judgments each pair of bulls $(\mathbf{v}, \mathbf{u})$ such that experts estimate that $\mathbf{v}$ carcass outperforms $\mathbf{u}$ 's carcass. We will show how it is possible, with a set of preference judgments, to learn a preference or assessment function.

Therefore, four experts of ASEAVA (Association of Breeders of Asturiana de los Valles) ordered groups of no more than 10 bulls according to the estimation of their carcass performance. We captured these orderings as a set of pairs of bulls where the first animal outperformed the second in experts' estimations. We call these pairs preference judgments, and in this way we collected 395 pairs. As was explained in the Introduction, we did not consider numerical estimations of carcass values since they were heavily biased by the so called batch effect. In the next sections we will spell out how we deal with this kind of data to produce a computable way to assess bulls.

On the other hand, once we had an assessment function, we needed to know how the assessments were changing with the age, since we wanted to adjust these amounts to a fixed age of $\mathbf{3 6 5}$ days. Thus, we collected a second set of data formed by 63 different bulls. They were used to study the growth of morphological measurements as well as the variation in time of the values returned by the assessment formula. Each bull (of $\mathbf{2 0 0}$ to $\mathbf{4 0 0}$ days old) was measured a minimum of $\mathbf{4}$ times and a maximum of $\mathbf{1 6}$ times, the total number of measurements of the 63 bulls was 587 .

\subsection{Numerical description of bulls}

We considered a set of possibilities in order to explore different combinations of numerical descriptions of bulls. We started with a set of measurements inspired in those recommended in guides for organizing linear scoring 
systems like those published periodically by ICAR. Additionally, we also included lengths like L4 (see Figure 1) that had been determined as very relevant to compute the assessment of carcasses, see (Díez et al. 2003). Thus, we considered the collection of measurements that can be obtained from lateral and rear views (see Figure 1), from each animal; additionally, we added the estimation of the curvature of their round profile on a scale of 1-5 (RP). To this set of variables, we added the sum of L5 and L4, since the whole measure of the top part could result useful independently of their components, and it is easier to obtain. Therefore, each bull was initially described by $\mathbf{8}$ lengths plus RP. Moreover, in order to facilitate the acquisition of measurements, the length L3 was assumed to be the hypotenuse of a right-angled triangle formed by L4+L5 and L2.

In general, to describe numerically the bulls, we will distinguish between raw input measures, which will be called variables, and features: those descriptors constructed from the original input variables. So, in order to try to describe faithfully the carcass merits, given that it is acknowledged that some volumes and areas can be very informative, we added all possible 2 and $\mathbf{3}$ dimensional data; and hence each animal was described by 165 features. Finally, we included all (non redundant) ratios in between the $\mathbf{8}$ lengths considered from each animal, since it is usually assumed that somehow harmonious proportions of body measurements are related to animals' performance. However, since we are interested in discovering features directly or indirectly proportional to expertise opinions, given a couple of lengths $\mathbf{L i}, \mathbf{L j}$ we only considered one of the two possible options: $\mathbf{L i} / \mathbf{L} \mathbf{j}$, and $\mathbf{L j} / \mathbf{L i}$. Therefore, we included $\mathbf{2 8}$ new features in other dataset with $\mathbf{1 9 3}$ features.

On the other hand, taking into account the complexity of obtaining the measurements from the lateral and rear views, we have considered the alternative of using only one stereo photograph from a zenithal point of view (see Figure 1), with the addition of the curvature of the RP. In this case, we do not have neither L6 nor L2; however, we observed that there is a high correlation between $\mathbf{L} 3$ and L4+L5, and then we can estimate L3 directly from L4+L5, and then we compute L2 using the right-angled triangle of these $\mathbf{3}$ lengths. Therefore, using this view we describe the animal by means of $\mathbf{7}$ lengths, and the curvature of the RP; finally, when we include the volumes and areas we have 120 features, and with the addition of ratios we have 141 features.

Therefore, we considered 4 options to describe bulls using vectors of 120, 141, 165, or 193 components respectively.

\subsection{Learning preferences}

Let us assume that

$$
\mathbf{P J}=\left\{\mathbf{v}_{\mathbf{i}} \text { outperforms } \mathbf{u}_{\mathrm{i}}: \mathbf{i}=\mathbf{1}, \ldots, \mathrm{n}\right\}
$$

is the set of orderings or preference judgments provided by experts about the estimation of performance of bull's carcasses. For ease of reference, let us assume that each bull is represented by a m-dimensional vector, that is, a point in $\mathbf{R}^{\mathbf{m}}$. Our aim is to find an ordering preserving (monotone) function $\mathbf{f}: \mathbf{R}^{\mathbf{m}} \rightarrow \mathbf{R}$ that will be called assessment or ranking function. In other terms, we look for an assessment $\mathbf{f}$ for $\mathrm{m}$-dimensional vectors such that maximize the probability of having $\mathbf{f}(\mathbf{v})>\mathbf{f}(\mathbf{u})$ whenever $\mathbf{v}$ represents a bull whose carcass outperforms the carcass of another bull represented by $\mathbf{u}$.

To simplify the problem, let us restrict the searching space to linear functions from $\mathbf{R}^{\mathbf{m}}$ to $\mathbf{R}$. Notice that those functions have the form

$$
\mathbf{f}_{\omega}: \mathbf{R}^{\mathrm{m}} \rightarrow \mathbf{R}, \mathbf{f}_{\omega}(\mathbf{x})=\langle\omega, \mathrm{x}>
$$

where $\omega$ is a weight vector, and $\langle\omega, \mathbf{x}>$ stands for the scalar product of $\omega$ and the input vector $\mathbf{x}$. Therefore, to find an assessment or ranking function from the data set of equation (1) is equivalent to find a vector $\omega$. The core idea (Herbrich et al., 2000; Joachims, 2002; Bahamonde et al. 2004) is that each preference judgment can be seen as an ordering constraint since

$$
\mathbf{f}_{\omega}(\mathbf{v})>\mathbf{f}_{\omega}(\mathbf{v}) \Leftrightarrow\langle\omega, v\rangle>\langle\omega, \mathbf{u}\rangle \Leftrightarrow\langle\omega, \mathbf{v}-\mathbf{u}\rangle>\mathbf{0} \Leftrightarrow \mathbf{f}_{\omega}(\mathbf{v}-\mathbf{u})>\mathbf{0}
$$

Thus, to maximize the probability of having (3) for all pairs in dataset of equation (1), using a margin maximization setting, formally, we must minimize:

$$
V(\omega, \xi)=\frac{1}{2}\|\omega\|^{2}+C \sum_{i=1}^{n} \xi_{i}
$$

subject to

$$
\forall \mathrm{i}=1, \ldots, \mathrm{n},\left\langle\omega, \mathrm{v}_{\mathrm{i}}\right\rangle \geq\left\langle\omega, \mathrm{u}_{\mathrm{i}}\right\rangle+1-\xi_{\mathrm{i}}, \xi_{\mathrm{i}} \geq 0
$$

where $\mathrm{C}$ is a parameter that allows trading-off margin size against training error. 
To implement the solution of this optimization problem, we can use a classification SVM (Vapnik, 1998). In fact, the problem of finding a linear assessment function can be viewed as a problem of finding an unbiased hyperplane (crossing the origin) that discriminate as positive all differences of preference judgments. This can be carried out using Joachims' SVM ${ }^{\text {light }}$ (Joachims, 1999). In the experiments reported below, we used this learning system with the default value of the parameter $\mathrm{C}$.

\subsection{A method to select a subset of features}

In the preceding section, we showed that it is possible to induce an assessment or ranking function by means of a classification SVM. Additionally, we can use an algorithm to order the set of features used to describe the training examples according to their usefulness to make an accurate classification rule. Then, we will use a model selection method to split the features list in order to obtain the most promising subset of features.

Given that we are using SVM to induce a linear model, it is possible to employ the algorithm called Recursive Feature Elimination (RFE) (Guyon et al. 2002) for ranking the features. This algorithm is an example of a backward feature elimination process. So, it starts with all possible features and removes one feature per iteration, the one with the smallest weight. Formally, RFE's criterion to remove features is the value of $\left(\omega_{i}\right)^{2}$, where $\omega_{\mathrm{i}}$ is the coefficient of the $\mathbf{i}$-th feature in the hyperplane equation induced by SVM. The details are shown in the algorithm of Figure 2

This algorithm let us obtain a ranked list $\mathbf{L}=\left(\mathbf{F}_{\mathbf{m}}, \mathbf{F}_{\mathbf{m}-\mathbf{1}}, \ldots, \mathbf{F}_{\mathbf{1}}\right)$ with $\mathbf{m}$ different feature subsets, where each $F_{i}$ is a subset with exactly $\mathbf{i}$ features. Due to the recursive elimination, features in a subset $\mathbf{F}_{\mathbf{i}}$ are optimal in some sense when considered together, although individually they could be less relevant than other features eliminated in a previous step. This is an interesting property of RFE since it takes into account possible relations between features, empowering the possibility of discovering useful groups of interrelated features that would be labeled as irrelevant if considered one by one. However, it should be noted that, given the greedy nature of RFE, $\mathbf{F}_{i}$ will not necessarily contain the $\mathbf{i}$ most useful features of the original feature set in order to achieve a higher accuracy.

Once obtained the ranked list of feature subsets, the next step shall be to select one of them. In general, we will be interested in a subset which lets the learner yield the best performance, in terms of accuracy; so we need to estimate the performance for every feature subset. This task can be accomplished by many different model selection techniques, for example, cross-validation (CV), a commonly used method that has been proved very reliable in many circumstances (Kohavi, 1995). Let us recall that in a k-fold cross-validation the data is split into $\mathrm{k}$ subsets of approximately the same size. Then, the learning algorithm is trained $\mathrm{k}$ times, each time leaving out one of the subsets from training, but using the left out subset to compute an accuracy estimate. Finally, the average of the $\mathrm{k}$ accuracy estimates is the estimation on unseen data.

Therefore, we determined the subset $\mathbf{F}_{\mathrm{r}}$ of features where

$$
r=\operatorname{argmax}\left(C V\left(F_{i}, T\right): i=1, \ldots, m\right)
$$

where CV stands for the accuracy estimation obtained by a 10 -fold cross validation for a set of features as $\mathbf{F}_{\mathbf{i}}$ and a training set as $\mathbf{T}$.

\subsection{Growth evolution of measurements and assessments}

Let us consider a set $\mathbf{B}$ of bovines. For each animal and each conformation variable (see section 2.2) we have recorded a number of measurements in different days. The whole data available can be represented by a kind of matrix $\mathbf{w}$ indexed by $\mathbf{B}$ and a set of measure days; that is to say $\mathbf{w}(\mathbf{a}, \mathbf{d})$ stands for the measurements of the animal a taken when it was $\mathbf{d}$ days old. The data so collected will be represented by

$$
\left\{\mathbf{w}(a, d): d \in I_{a}, a \in B\right\}
$$

where $\boldsymbol{l}_{\mathbf{a}}$ is the set of dates of measurements for animal $\mathbf{a}$.

A first attempt to generalize these data consists in computing one regression function $\mathbf{f}_{a}$ for each animal. Then, if we want to be able to estimate the features of a new animal (not in $\mathbf{B}$ ), we have two options. If we have obtained reasonably good approximations for each animal with the family $\left(\mathbf{f}_{\mathbf{a}}: \mathbf{a} \in \mathbf{B}\right)$, and these family of functions belong to the same class, say linear for instance, in that case we can try to induce a new regressor of the same class for the new animal. Unfortunately, faithful regressors need a large collection of observations, and this requires time, what it is not acceptable. Additionally, the acquisition of data is costly and in general a risky task. 
The second option consists in trying to generalize somehow the data collected in matrix $\mathbf{w}$ to obtain a kind of universal function able to estimate the variable values of any animal at any time. It is not clear how to do this; and, on the other hand, it is necessary to consider individual differences: in some cases they are really important. But if we were able to devise a method to take advantage of all $\mathbf{w}(\mathbf{a}, \mathbf{d})$ at the same time, we can wait an improved generalization performance, since the number of such data in practice is usually high.

The method that we are proposing in this paper emphasizes the role of the trajectories followed in the space of measurements and days $(\mathbf{W} \times \mathbf{D})$ by the successive measurements of the animals. Figure 3 shows the trajectories followed by the assessments of $\mathbf{3}$ bulls computed every $\mathbf{1 5}$ days at ages from below $\mathbf{2 0 0}$ days until more than $\mathbf{4 0 0}$ days. The core assumption that we are going to use is that these trajectories are parallel. In this way we can develop a regression method that can produce a general growth model with a high correlation coefficient.

The assumption of parallelism gives rise to an estimation of $\mathbf{w}(\mathbf{a}, \mathbf{d})$ as

$$
w(a, d) \approx f(d)+k(a) \text {. }
$$

Intuitively, $\mathbf{f ( d )}$ copes with the shape of the trajectory, while $\mathbf{k}(\mathbf{a})$ is a constant of each animal and represents the height of the trajectory. To find the expressions of $\mathbf{f}$ and the value of $\mathbf{k}(\mathbf{a})$ for each animal, let us consider that if all available bulls were measured with $\mathbf{d}_{0}$ days old, then we could assume that

$$
\mathrm{k}(\mathrm{a}) \approx \mathbf{w}\left(\mathrm{a}, \mathrm{d}_{0}\right)-\mathrm{f}\left(\mathrm{d}_{0}\right)
$$

If we have more common measurement days, say the days included in a set $\mathbf{I}$, we can improve the approximation of the constant $\mathbf{k}(\mathbf{a})$ for each animal by the following average

$$
k(a)=\frac{1}{\|} \sum_{i \in 1} w\left(a, d_{i}\right)-\frac{1}{\|} \sum_{i=1} f\left(d_{i}\right)
$$

Therefore, equation (7) can be rewritten as

$$
w(a, d)-\frac{1}{\|} \sum_{i=1} w\left(a, d_{i}\right) \approx f(d)-\frac{1}{\|} \sum_{i=1} f\left(d_{i}\right)=g(d)
$$

In other words, the difference between the experimental measurements and the average on a given common set of ages is an amount that only depends on the age. If the dates of weights are not exactly the same, we can approximate the values by means of interpolation. Hence, we can assume that there are a set $\mathbf{I}$ of ages with measures for all bulls in B. Therefore, on the assumption that evolutions follow parallel trajectories, we can learn a model of the shape of them from the following dataset:

$$
\left\{w(a, d)-\frac{1}{\|} \sum_{i=1} w\left(a, d_{i}\right): d \in I_{a}, a \in B\right\}
$$

Thus, if $\mathbf{g}$ is a model found by regression from this dataset, then, for each animal a the measurement value at age $\mathbf{d}$ is predicted by

$$
w(a, d)=g(d)+\frac{1}{\|} \sum_{i \in 1} w\left(a, d_{i}\right)
$$

However, equation (12) is a not practical at all since the average measurement over dates in I is not available. Thus we must estimate this average, a constant of the animal. So, if we know w(a, d') for some age d', we simply subtract $\mathbf{g}\left(\mathbf{d}^{\prime}\right)$ from $\mathbf{w}\left(\mathbf{a}, \mathbf{d}^{\prime}\right)$. In general, if we have a set $\left\{\mathbf{w}\left(\mathbf{a}, \mathbf{d}_{\mathbf{j}}\right): \mathbf{j} \in \mathbf{J}\right\}$ of measurements available, we estimate

$$
\frac{1}{\|} \sum_{i=1} w\left(a, d_{i}\right) \approx \frac{1}{|J|} \sum_{j \in J}\left(w\left(a, d_{j}\right)-g\left(d_{j}\right)\right)
$$

Finally, the estimated evolution of measurements for bull $\mathbf{a}, \mathbf{d}$ days old, is given by:

$$
w(a, d)=g(d)+\frac{1}{|J|} \sum_{j \in J}\left(w\left(a, d_{j}\right)-g\left(d_{j}\right)\right)
$$

\section{Results and discussion}

\subsection{Experimental results for learning assessments}

Using the data described above, we have conducted some experiments to obtain an assessment formula coherent with the set of 395 preference judgments available. As discussed in section 2.2, we considered 4 
options to describe bulls using vectors of $120,141,165$, or 193 components respectively. Each option gave rise to a dataset of $\mathbf{3 9 5}$ differences named 'bulls-' followed by the number of components. Let us highlight that the ratio between the number of features and the number of training examples is not a problem for SVM. In fact these Machine Learning algorithms have been successfully used in fields where the number of examples was a few tens described by thousands of features; see, for instance (Guyon et al. 2002). This skill of SVM opens new possibilities in Livestock research, since it will be feasible to handle more complex descriptions of animals in prediction tasks.

With this datasets we estimated the accuracy using a 10-fold cross validation as it was explained in section 2.4. In order to test the selection of features, we used two different learning approaches. First we used a SVM preceded by the feature selection algorithm described in section 2.4. Since the selection is part of the learning algorithm, in each fold of the cross validation we used separated sets for selection during training stage and testing; therefore, the number of features selected is different in each fold. Additionally, for the sake of completion we used a SVM without any feature selection. The scores so obtained are reported in Table 1, see (Bahamonde et al. 2004).

In all cases the method that selects the features to induce an assessment function outperforms the accuracy found by SVM without any filter. On the other hand the accuracies achieved filtering first the set of features are quite similar. Thus, the selection of the assessment function can be based on the reduction of variables. Hence, we prefer the function learned from bulls-141 given that it is only $\mathbf{0 . 0 1}$ below the accuracy of the best (bulls193), but the average number of features used is only 3.9 against 5.7. Moreover, bulls-141 uses only measurements that can be acquired from a zenithal stereo view.

The final assessment function learned using all data of bulls-141 dataset uses the following variables: L4, L5, L7, and RP (see Figure 1).

\subsection{Growth of assessments}

Using the dataset of 587 measurements described in section 2.1, and following the procedure explained in section 2.6, we can follow two ways to model the growth of the assessment scores. First, we can try to model the evolution of each of the variables included in the assessment formula. However, this approach has some problems. In Table 2, we show the correlation coefficients achieved for each of the variables; in all cases the model has correlation higher than $\mathbf{0 . 9}$, except in the case of the curvature of the round profile (RP) where we only can reach a poor $\mathbf{0 . 4 9 7}$. The reason is that $\mathbf{R P}$ is mostly constant with some small increments in some animals.

On the other hand we can try to model the growth of assessment scores. In this case the correlation is $\mathbf{0 . 9 0 6}$, what ensures good predictions of the growth of the assessments. In Figure 4 we show the cloud of points used for obtaining the model and the function that depicts the shape of the growth. This function means that an accurate prediction for the assessment growth is $\mathbf{3 . 5 7}$ points each $\mathbf{1 0 0}$ days. Formally, the equation to estimate the assessment of animal $\mathbf{a}$ at age $\mathbf{3 6 5}$ days, knowing the assessments obtained in dates of $\left\{\mathbf{d}_{\mathbf{j}}: \mathbf{j} \in \mathbf{J}\right\}$, is given by

$$
\begin{aligned}
& \operatorname{Assess}(\mathrm{a}, 365)=(0.0357 \times 365-9.07)+ \\
& \qquad \begin{aligned}
\frac{1}{|J|} \sum_{j \in J}\left(\operatorname{Assess}\left(\mathrm{a}, \mathrm{d}_{\mathrm{j}}\right)-\left(0.0357 \mathrm{~d}_{\mathrm{j}}-9.07\right)\right) \\
=\frac{1}{|J|} \sum_{\mathrm{j} \in J} \operatorname{Assess}\left(\mathrm{a}, \mathrm{d}_{\mathrm{j}}\right)+0.0357\left(365-\frac{1}{|J|} \sum_{\mathrm{j} \in J} \mathrm{~d}_{\mathrm{j}}\right)
\end{aligned}
\end{aligned}
$$

\subsection{The final formula for assessing bulls}

Taking into account the results showed in previous sections, the final assessment of bulls can be carried out using the function detailed in Figure 5. The inputs are the lengths L4, L5, and L7 (see Figure 1) in centimeters, and the curvature of the round profile (RP) in a scale 1-5. Additionally, we require the age of the bull in days when it was measured.

The first expression to compute the variable assess reflects that the assessment depends on 3 features: the quotient L7/L2; the product of L4, L5, and L7; and the round profile. These features are first normalized; that is, 
according to maximum and minimum values observed, they are transformed into values from $\mathbf{0}$ to $\mathbf{1}$. Then these amounts are weighted by coefficients found by the SVM. Notice that the first two features have greater influence than the round profile.

Finally, assess is rescaled to obtain a score from $\mathbf{0}$ to $\mathbf{1 0 0}$ points, as it was traditionally used by the Association of Breeders. The last operation is performed to return an adjusted 365-days score of assess. If we had colleted a set of measurements in different days, then we would have applied equation (15).

\section{Implications}

We have shown the steps followed to develop an assessment procedure for a specialized beef breed: Asturiana de los Valles. The scores aim to order animals according to their carcass values established at a constant age of 365 days. The procedure described here has two main characteristics.

First, it is a repeatable procedure since it is based on measurements in centimeters of only 3 lengths of the animals plus an easy appreciation of the curvature of the round profile on a scale of 1-5. These measurements can be obtained manually or using digital photographs.

Second, the assessment has used the ordering criteria expressed by the experts of the association of breeders according to the market conditions of the breed. To transform experts' opinions into a computable formula, we used Artificial Intelligence tools based on Support Vector Machines that allow us both to learn the preferences of the market and to select the variables required to input an assessment function.

\section{Acknowledgements}

The research reported in this paper is supported in part under the grant TIN2005-08288 from the Spanish Ministerio de Educación y Ciencia. The authors would like to acknowledge the collaboration of the experts of the Association of Breeders (ASEAVA) during the acquisition of data stage.

\section{References}

1. Bahamonde, A.; Bayón, G. F.; Díez, J.; Quevedo, J. R.; Luaces, O.; Del Coz, J. J.; Alonso, J.; Goyache, F.. Feature subset selection for learning preferences: a case stud y. Proceedings of the 21st International Conference on Machine Learning, ICML 2004, Banff, Canada, (2004) 49-56

2. Del Coz, J. J.; Bayón, G. F.; Díez, J.; Luaces, O.; Bahamonde, A.; Sañudo, C.. Trait sele ction for assessing beef meat quality using non -linear SVM. Proceedings of the Eigh teenth Annual Conference on Neural Information Processing Systems (NIPS 2004). Vancouver, British Columbia, Canada, (2004)

3. Díez, J.; Bahamonde, A.; Alonso, J.; López, S.; del Coz, J. J.; Quevedo, J.; Ranilla, J.; Luaces, O.; Álv arez, I.; Royo, L.; Goyache, F. (2003). Artificial intelligence techniques point out differences in classification performance between light and standard bovine carcasses. Meat Science, 64(3), pp. 249-258

4. Díez, J.; Del Coz, J. J.; Bahamonde, A.; Sañudo, C.; Olleta, J. L.; Macie, S.; Campo, M. M.; Panea, B.; Albertí, P. (2006): Identifying market segments in beef: Breed, slaughter weight and ageing time implications. Meat Science, 74(4), pp. 667675

5. Díez, J.; Del Coz, J. J.; Sañudo, C.; Albertí, P.; Bahamond e, A. (2005). A Kernel Based Method for Discovering Market Segments in Beef Meat. Proceedings of the 16th European Conference on Machine Learning - 9th European Conference on Principles and Practice of Knowledge Discovery in Databases, ECML/PKDD'2005, Porto, Portugal, pp. 462-469

6. Guyon, I., Weston, J., Barnhill, S., \& Vapnik, V. (2002). Gene selection for cancer classification using support vector machines. Machine Learning, 46, 389-422.

7. Herbrich, R.; Graepel, T.; and Obermayer, K.: Large margin rank boun daries for ordinal regression. In A. Smola, P. Bartlett, B. Scholkopf, and D. Schuurmans, editors, Advances in Large Margin Classifiers, pages 115 -132. MIT Press, Cambridge, MA, (2000)

8. ICAR (International Committee for Animal Recording). (2005). Internatio nal Agreement of Recording Practices. ihttp://www wicar.org!

9. Joachims, T.. Making large -Scale SVM Learning Practical. Advances in Kernel Methods - Support Vector Learni ng, B. Schölkopf, C. Burges and A. Smola (ed.), MIT-Press, (1999)

10. Joachims, T.: Optimizing search engines using clickthrough data. In: Proceedings of the ACM Conference on Knowledge Discovery and Data Mining (KDD) (2002) 
11. Koch, R.M., Cundiff, L.V., Gregory, K.E., Van Vleck, L.D. (2004). Genetic Response to Selection for Weaning Weight Or Yearling Weight Or Yearling Weight and Muscle Score in Hereford Cattle: Efficiency of Gain, Growth, and Carcass Characteristics. Journal of Animal Science, 82:668-682.

12. Kohavi, R. (1995). A study of cross -validation and bootstrap for accuracy estimation and model selection. Procs. of the IJCAI (pp. 1137-1145).

13. Nephawe, K.A., Cundiff, L.V., Dikeman, M.E., Crouse, J.D., Van Vleck, L.D. (2004). Genetic Relationships Between Sex-Specific Traits in Beef Cattle: Mature Weight, Weight Adjusted for Body Condition Score, He ight and Body Condition Score, Height and Body Condition Score Measurement of Cows and Carcass Traits of Their Steer Relat ives. Journal of Animal Science, 82:647-653.

14. Piedrafita J., Quintanilla R., Sañudo C., Olleta J.L., Campo M.M., Panea B., Renand G., T urin F., Jabet S., Osoro K., Oliván M.C., Noval G., García P., García M.D., Cruz -Sagredo R., Oliver M.A., Gispert M., Serra X., Espejo M., García S., López M., Izquierdo M. (2003). Carcass quality of 10 beef cattle breeds of the Southwest of Europe in thei $r$ typical production systems. Livestock Production Science, 82, 1-13.

15. Vapnik, V. (1998). Statistical learning theory. John Wiley. 


\begin{tabular}{c|cc|c}
\hline & \multicolumn{2}{|c|}{ Filtering features } & All features \\
Dataset & $\%$ Acc & \#Feat & \%Acc \\
\hline bulls-120 & $96.46 \pm 3.03$ & $6.40 \pm 3.47$ & $94.17 \pm 2.79$ \\
bulls-141 & $96.69 \pm 2.82$ & $3.90 \pm 1.45$ & $94.68 \pm 2.89$ \\
bulls-165 & $96.20 \pm 3.45$ & $4.50 \pm 1.28$ & $94.42 \pm 2.24$ \\
bulls-193 & $96.70 \pm 2.30$ & $5.70 \pm 1.19$ & $94.68 \pm 2.41$ \\
\hline
\end{tabular}

Table 1. Classification accuracies estimated by a 10-fold cross validation using a dataset of 395 preference judgments about 91 bulls

\begin{tabular}{c|cc}
\hline Measurement & degree & $\mathrm{R}$ \\
\hline L4 (loin length) & 3 & 0.914 \\
L5 (rump length) & 2 & 0.914 \\
L7 (thighs width) & 2 & 0.941 \\
RP (round profile) & 1 & 0.497 \\
\hline
\end{tabular}

Table 2. Description of models obtained (with a stepwise regression for the successive powers of the age) for the growth of variables included in the assessment formula discussed in section 3.1. The number of bulls considered was 63 ; each bull was measured from $\mathbf{4}$ to $\mathbf{1 6}$ times; the total number of measurement considered was $\mathbf{5 8 7}$

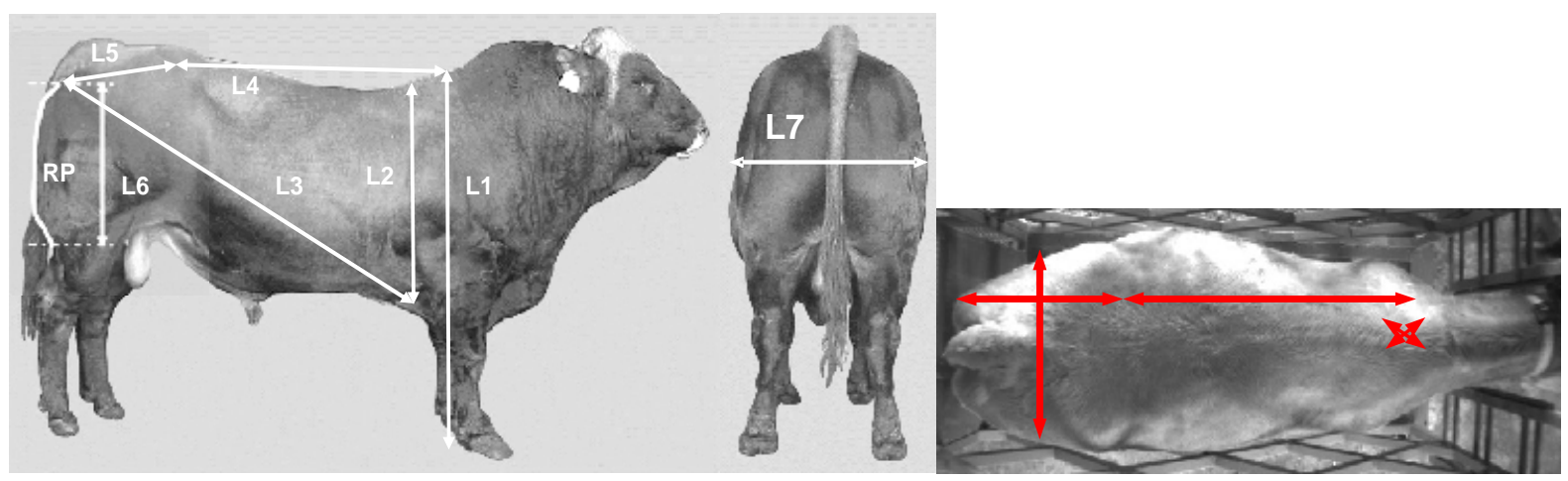

Fig. 1. Digital images used to acquire the measurements used in this paper. The leftmost two images, lateral and rear views, show 7 lengths plus the round profile (RP). The right image is a zenithal view, one of the two stereo images that can be used instead of the other views 
Function SVM-RFE(T, fs): A list of feature subsets

\section{BEGIN}

$I^{*} \mathrm{~T}$ : Set of training examples; each example is described by a vector of feature values $(x)$ and its class $(y)$

fs: Set of features describing each example in $\mathrm{T}$;

L: Ordered list of feature subsets; each subset contains the

$F_{m}=f s$; remaining features at every iteration */

$\mathrm{L}=\left[\mathrm{Fm}_{\mathrm{m}}\right]$

for $\mathrm{j}=\mathrm{m}$ downto 2 do

$\alpha=\operatorname{SVM}(T)$;

$/ / \mathrm{m}$ is the number of features

// Initially, one subset with all the features

$\omega=\sum_{\mathrm{k}} \alpha_{\mathrm{k}} \mathrm{y}_{\mathrm{k}} \mathrm{x}_{\mathrm{k}}$

// Train SVM

$r=\operatorname{argmin}\left(\left(\omega_{i}\right)^{2}: i=1, \ldots,\left|F_{i}\right|\right) ; / /$ The smallest ranking criterion

$F_{j-1}=F_{j} \backslash f_{r}$;

$L=L+F_{j-1}$;

$T=\left\{X^{\prime}: x^{\prime}\right.$
end for

$T=\left\{x_{i}^{\prime}: x_{i}^{\prime}\right.$ is $x_{i} \in T$ with $f_{r}$ removed $\}$

// Remove $r$-th feature from $\mathrm{Fj}_{\mathrm{j}}$

// Add the subset of remaining features

// Remove $r$-th feature from examples in $\mathrm{T}$

return $(\mathrm{L})$;

END

// Return the ordered list $L$ of feature subsets

Fig. 2. Pseudo code of the algorithm (SVM-RFE) used to rank the set of features available

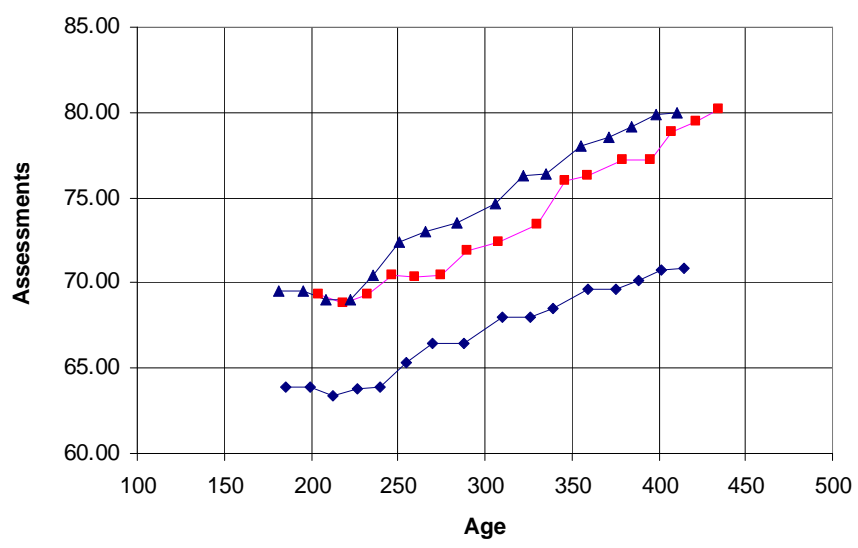

Fig. 3. Evolution of $\mathbf{1 6}$ assessments of $\mathbf{3}$ bulls during the measuring stage 


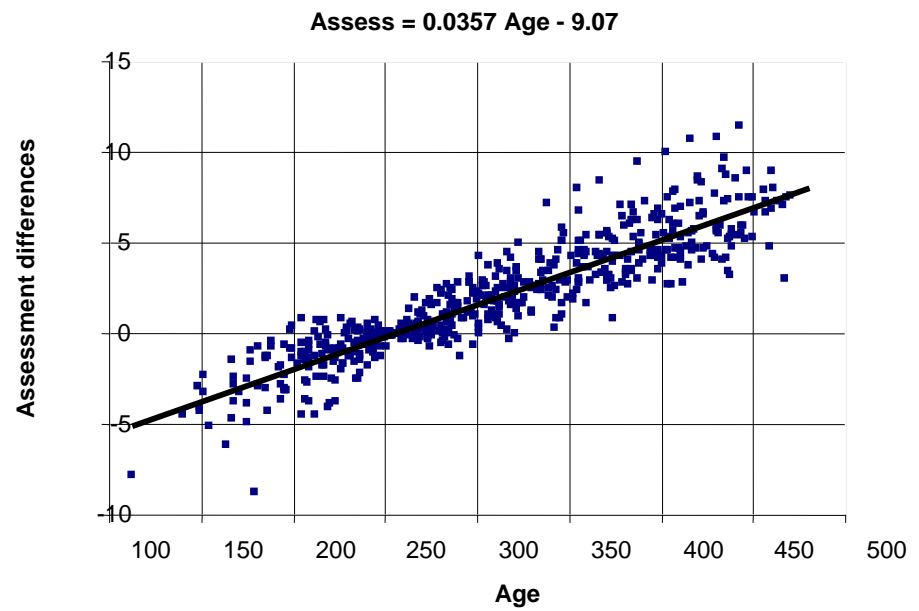

Fig. 4. Linear relation between assessment differences and the age of animals given in days. The number of measurements used was $\mathbf{5 8 7}$. The correlation coefficient (R) is $\mathbf{0 . 9 0 6}$

Function Assessment (L4, L5, L7, RP, d): the assessment of the bull BEGIN

$I^{*} \quad$ L4, L5, L7: Lengths of the bull according to Figure 1;

RP: Curvature of the round profile of the bull codified between 1 and 5

$\mathrm{d}$ : Age, in days, of the bull

$\mathrm{L} 3=(\mathrm{L} 4+\mathrm{L} 5) * 1.1+6.23 ; \quad / /$ experimental estimation of $\mathrm{L} 3$

$\mathrm{L} 2=\sqrt{\mathrm{L}^{2}-(\mathrm{L} 4+\mathrm{L} 5)^{2}}$

Assess $=2.33\left(\frac{\mathrm{RP}-0.5}{5}\right)+3.43\left(\frac{\mathrm{L} 7 / \mathrm{L} 2^{-0.35}}{0.2}\right)+3.35\left(\frac{\mathrm{L} 7 \mathrm{~L} 4 \mathrm{~L} 5-661 * 10^{2}}{132 * 10^{3}}\right)$

Assess $=3.74$ Assess $+67.1 \quad / /$ experimental parameters for rescaling

Assess $=$ Assess $+0.0357(365-d) \quad$ // adjusted 365-days

return (Assess);

END

Fig. 5. Algorithm to compute the assessment of a bull on a scale of $\mathbf{0 - 1 0 0}$ points adjusted $\mathbf{3 6 5}$-days. Input $\mathbf{d}$ is the age (in days) of the bull when it was measured 